\title{
Wave Functions for SU(2) Hamiltonian Lattice Gauge Theory
}

\author{
Matteo Beccaria \\ Dipartimento di Fisica dell'Università di Lecce, I-73100, Italy, \\ Istituto Nazionale di Fisica Nucleare, Sezione di Lecce
}

\begin{abstract}
We study four dimensional $\mathrm{SU}(2)$ lattice gauge theory in the Hamiltonian formalism by Green's Function Monte Carlo methods. A trial ground state wave function is introduced to improve the configuration sampling and we discuss the interplay between its complexity and the simulation systematic errors. As a case study, we compare the leading strong coupling approximation and an improved 4 parameters wave function with $1 \times 1$ and $1 \times 2$ plaquette terms. Our numerical results favors the second option.
\end{abstract}

PACS numbers: 11.15.Ha, 12.38.Gc 


\section{INTRODUCTION}

In the study of Lattice Gauge Theory, a major alternative to the usual Lagrangian formulation is the Hamiltonian version of Kogut-Susskind [1]. This approach is relevant both for theoretical reasons, e.g. universality checks, and for practical purposes because it seems the natural choice for static problems, like spectroscopy [2].

An important feature of the Hamiltonian formulation is that it can exploit analytical approximations of the ground state wave function to improve the numerical evaluation of physical quantities [3]. This well known procedure is called Importance Sampling [4]. However, even if an accurate wave function is certainly welcome, on the other hand it can also require expensive calculations. A careful analysis is therefore necessary to understand the interplay between accuracy and performance and to determine which is the optimal complexity of the trial wave function.

A recent investigation of Hamiltonian methods in the numerical study of non Abelian gauge theories can be found in [5] where the $\mathrm{SU}(2)$ model is studied in $2+1$ dimensions. Here, we focus mainly on the wave function issue and study the $3+1$ dimensional $\mathrm{SU}(2)$ pure gauge lattice theory by a particular Green's Function Monte Carlo (GFMC) [6] algorithm that computes statistical averages over an ensemble with a fixed number of random walkers [7]. This algorithm has the desirable feature of allowing a particularly simple analysis of the systematic errors.

On general grounds and motivated by strong coupling expansions [8] we consider trial wave functions which are expressed by combinations of gauge invariant Wilson loops with unknown coefficients a. Two main issues are addressed: the cost of computing large loops and the practical simultaneous optimization of many parameters a. About the latter, we test a recently proposed algorithm for the adaptive optimization of $\mathbf{a}$. It is a method that has been successfully applied to the study of a very simple system with U(1) gauge symmetry 9 9 and that deserves a more detailed analysis on a realistic test-bed like the non Abelian $\mathrm{SU}(2)$ four dimensional model.

As we shall see, the most serious systematic error is related to the finite population size, namely the number of walkers. The extrapolation to an infinite population is difficult and much easier when an improved wave function is used. Working with a four parameter trial function, we present results for the extrapolated quantities and study the scaling of the string tension comparing our results with similar Lagrangian calculations.

The plan of the paper is the following: in Sects. II, III and IV, we present the GFMC algorithm in its general form for $\mathrm{SU}(\mathrm{N})$ lattice gauge theories with Importance Sampling, fixed number of random walkers and adaptive optimization of the trial wave function. In Sec. $\mathrm{V}$, we apply the proposed algorithm to the SU(2) model in four dimensions and discuss the numerical results.

\section{REVIEW OF GFMC FOR SU(N) GAUGE THEORY}

In this Section, we recall the Feynman-Kac-Nelson formula for the matrix elements of the evolution operator associated with the SU(N) Kogut-Susskind Hamiltonian. This formula

provides probabilistic representations of several interesting quantities. The GFMC algorithm is just one of its numerical implementations. 
Let $U=\left\{U_{l}\right\}$ be the set of group elements, one for each link $l$; let $E=\left\{E_{l}^{\alpha}\right\}$ be the associated electric field defined by

$$
\left[E_{l}^{\alpha}, U_{l^{\prime}}\right]=T^{\alpha} U_{l} \delta_{l, l^{\prime}}
$$

where $\left\{T^{\alpha}\right\}, \alpha=1, \ldots, N^{2}-1$ are $\mathrm{SU}(\mathrm{N})$ generators. Finally, let $V(U)$ be a gauge invariant real potential. The Kogut-Susskind Hamiltonian for $\mathrm{SU}(\mathrm{N})$ lattice gauge theory is then $H_{K S}=g^{2} H$ where $g$ is the gauge coupling and

$$
H=H_{0}+V(U), \quad H_{0}=\frac{1}{2} E^{2} \equiv \frac{1}{2} \sum_{\alpha=1}^{N^{2}-1} \sum_{l}\left(E_{l}^{\alpha}\right)^{2} .
$$

For the Wilson action, $V(U)$ is proportional to the sum of the smallest $1 \times 1$ plaquette loops $U_{p}$ :

$$
V(U)=-\frac{1}{g^{4}} \sum_{p} \operatorname{Tr}\left(U_{p}+U_{p}^{\dagger}\right),
$$

but the present discussion holds for a general $V(U)$.

The matrix elements of the Euclidean evolution operator $\exp (-t H)$ in the basis of $U_{l}$ eigenstates admit the small $t$ expansion

$$
D\left(U^{\prime \prime}, U^{\prime}, \varepsilon\right)=\left\langle U^{\prime \prime}\left|e^{-\varepsilon H}\right| U^{\prime}\right\rangle=D_{0}\left(U^{\prime \prime}, U^{\prime}, \varepsilon\right) e^{-\varepsilon V\left(U^{\prime}\right)}+\mathcal{O}\left(\varepsilon^{2}\right),
$$

where the factor

$$
D_{0}\left(U^{\prime \prime}, U^{\prime}, \varepsilon\right)=\left\langle U^{\prime \prime}\left|e^{-\varepsilon H_{0}}\right| U^{\prime}\right\rangle,
$$

can be interpreted as a probability density for the random transition $U^{\prime} \rightarrow U^{\prime \prime}$ due to the relations:

$$
D_{0}\left(U^{\prime \prime}, U^{\prime}, \varepsilon\right)>0 \quad \text { and } \quad \int D_{0}\left(U^{\prime \prime}, U^{\prime}, \varepsilon\right) d U^{\prime \prime}=1,
$$

( $d U$ is the $\mathrm{SU}(\mathrm{N})$ Haar measure). Inserting intermediate states between $U^{\prime \prime}$ and $U^{\prime}$, we obtain as usual

$$
D\left(U^{\prime \prime}, U^{\prime}, t\right)=\int\left\langle U^{\prime \prime}\left|e^{-(t /(N+1)) H}\right| U_{N}\right\rangle \cdots\left\langle U_{1}\left|e^{-(t /(N+1)) H}\right| U^{\prime}\right\rangle d U_{1} \cdots d U_{N},
$$

and taking the $N \rightarrow \infty$ limit we recover the Feynman-Kac-Nelson path integral representation of $D\left(U^{\prime \prime}, U^{\prime}, t\right)$ :

$$
D\left(U^{\prime \prime}, U^{\prime}, t\right)=\int_{U(0)=U^{\prime}, U(t)=U^{\prime \prime}} \mathcal{D} U(t) e^{-\int_{0}^{t} d \tau V(U(\tau))},
$$

where the measure $\mathcal{D} U(t)$ stands for the $N \rightarrow \infty$ limit of the average Eq. (2.7) over random group polygonals $\left(U_{0}=U^{\prime}, U_{1}, \ldots, U_{N}, U_{N+1}=U^{\prime \prime}\right)$.

The potential $V(U)$ fluctuates along the path $U(t)$ and determines the error on the estimates of physical quantities. To see how this happens, let us consider in this framework 
the calculation of the ground state energy $E_{0}$. The simplest procedure is to compute the limit

$$
E_{0}=\lim _{t \rightarrow+\infty} E\left(t, U^{\prime}\right)
$$

where

$$
E\left(t, U^{\prime}\right)=-\frac{d}{d t} \log \int D\left(U^{\prime \prime}, U^{\prime}, t\right) d U^{\prime \prime}, \quad \quad U^{\prime} \text { arbitrary. }
$$

It is easy to check that

$$
E\left(t, U^{\prime}\right)=\frac{\int_{U(0)=U^{\prime}} \mathcal{D} U(t) e^{-\int_{0}^{t} d \tau V(U(\tau))} V(U(t))}{\int_{U(0)=U^{\prime}} \mathcal{D} U(t) e^{-\int_{0}^{t} d \tau V(U(\tau))}} \equiv\langle V(U(t))\rangle,
$$

where $\langle\cdot\rangle$ is the average over free trajectories on the group manifold weighted by the exponential term. Hence, the fluctuations of $V$ control the noise in any estimator of $E_{0}$. Other problems in the actual numerical evaluation of $\langle V\rangle$ can be discussed separately and will be considered in the next Section.

The introduction of a trial wave function is a clever way to reduce the fluctuations of $V$. To this aim, one considers a positive function $\Psi_{0}(U)=\exp F(U)$ and the unitarily equivalent Hamiltonian

$$
\widetilde{H}=\Psi_{0}\left[H_{0}+V(U)\right] \Psi_{0}^{-1}
$$

which turns out to be

$$
\begin{aligned}
\widetilde{H} & =\widetilde{H_{0}}+\widetilde{V}, \\
\widetilde{H_{0}} & =\frac{1}{2} E^{2}+i E \cdot \nabla F, \\
E \cdot \nabla F & \equiv \sum_{l, \alpha} E_{l}^{\alpha} \nabla_{\alpha, l} F \\
\widetilde{V} & =V-\frac{1}{2} \sum_{\alpha, l}\left(\nabla_{\alpha, l} F\right)^{2}-\frac{1}{2} \sum_{\alpha, l} \nabla_{\alpha, l}^{2} F,
\end{aligned}
$$

where $\nabla_{\alpha, l}$ is the group invariant derivative on $\mathrm{SU}(\mathrm{N})$ associated with link $l$. The following approximate factorization of the $\widetilde{H}$ propagator holds

$$
\widetilde{D}=\widetilde{D_{0}} e^{-\varepsilon \widetilde{V}}+\mathcal{O}\left(\varepsilon^{2}\right)
$$

where $\widetilde{D}_{0}$ is the kernel of $\exp \left(-\varepsilon \widetilde{H_{0}}\right)$ and satisfies the fundamental relations

$$
\widetilde{D}_{0}\left(U^{\prime \prime}, U^{\prime}, \varepsilon\right)>0 \quad \text { and } \quad \int \widetilde{D}_{0}\left(U^{\prime \prime}, U^{\prime}, \varepsilon\right) d U^{\prime \prime}=1
$$

(the important point is that $\widetilde{H_{0}}$ is normal ordered with all the $E$ operators acting from the left). As in the $F \equiv 0$ case, the finite time propagator $\widetilde{D}$ may be expressed in terms of a weighted average 


$$
\widetilde{D}\left(U^{\prime \prime}, U^{\prime}, t\right)=\int_{U(0)=U^{\prime}, U(t)=U^{\prime \prime}} \widetilde{\mathcal{D}} U(t) e^{-\int_{0}^{t} d \tau \widetilde{V}(U(\tau))},
$$

where $\widetilde{\mathcal{D}} U$ is the measure determined by the form of $\widetilde{D}$ at infinitesimal times. The explicit first-order discrete construction of the paths is done by choosing a time step $\varepsilon$ and updating $U_{n} \rightarrow U_{n+1}$ according to the rule

$$
U_{n+1}=U_{R}(\varepsilon) \cdot U_{D}\left(\varepsilon, U_{n}\right) \cdot U_{n},
$$

where $U_{R}(\varepsilon)$ is a random $\mathrm{SU}(\mathrm{N})$ element distributed according to the heat kernel [10, [1] at time $\varepsilon$ and $U_{D}(\varepsilon, U)$ is the drift

$$
U_{D}(\varepsilon, U)=\exp (i \varepsilon T \cdot \nabla F(U)) .
$$

When $\Psi_{0}$ happens to be an exact eigenstate of $H$ with eigenvalue $E$, we have $\widetilde{V} \equiv E$ and the formula

$$
E(t)=\langle\tilde{V}(U(t))\rangle=E,
$$

provides the correct eigenvalue with zero variance, namely no statistical error. In other words, if we require $\widetilde{V}$ to be a constant, then the problem is completely equivalent to solve the Schrödinger equation for the Kogut-Susskind Hamiltonian. In the following, we shall try to obtain a constant $\widetilde{V}$ at least in some approximate sense.

In the above discussion we focused on the ground state energy which is the simplest observable. A more general case is that of ground state matrix elements of diagonal operators in the basis of the link variables $U$ :

$$
\langle\Omega\rangle=\langle 0|\Omega(U)| 0\rangle,
$$

where $|0\rangle$ denotes the ground state of $\widetilde{H}$. They can be evaluated by computing

$$
\langle\Omega\rangle=\lim _{t_{2} \rightarrow+\infty} \lim _{t_{1} \rightarrow+\infty} \Omega\left(t_{2}, t_{1}, U^{\prime}\right),
$$

where

$$
\Omega\left(t_{2}, t_{1}, U^{\prime}\right)=\frac{\int_{U(0)=U^{\prime}} \mathcal{D} U(t) e^{-\int_{0}^{t_{1}+t_{2}} d \tau V(U(\tau))} V\left(U\left(t_{1}\right)\right)}{\int_{U(0)=U^{\prime}} \mathcal{D} U(t) e^{-\int_{0}^{t_{1}+t_{2}} d \tau V(U(\tau))}} .
$$

A similar formula can be also devised for many time correlation functions of operators in the Heisenberg representation.

\section{STOCHASTIC RECONFIGURATION}

In this Section, we discuss the actual calculation of the above mentioned averages over trajectories. Here, the trial wave function $\Psi_{0}$ will not play any role.

A naive implementation of the Feynman-Kac-Nelson representation generates group trajectories according to the measure $\mathcal{D} U$ and weights them with a potential dependent weight. 
Such weight increases or vanishes exponentially fast with respect to the trajectory length $t$. This is a serious numerical problem referred to as the "exploding variance problem". The name stems from the fact that the weight variance of a collection of random walkers explodes as $t \rightarrow+\infty$ overwhelming the numerical capabilities of any machine [12].

A possible solution to this problem consists in killing and cloning the random walkers with definite rules in order to delete the walkers with low weights and duplicate the others. The main disadvantage is that the size of the walker population varies in time and may become unstable.

The recently proposed Stochastic Reconfiguration Algorithm [7] is a simple implementation of the killing and branching idea, but with the desirable feature of dealing with a population with a fixed number of walkers. Its coding is therefore much simpler.

To this aim, a finite collection of $K$ walkers, an ensemble, is introduced

$$
\mathcal{E}=\left\{\left(U^{(n)}(t), \omega^{(n)}(t)\right)\right\}_{1 \leq n \leq K},
$$

where the weights $\omega^{(n)}$ are defined by

$$
\omega^{(n)}(t)=\exp -\int_{0}^{t} d \tau V\left(U^{(n)}(\tau)\right)
$$

The variance of the weights over the ensemble $\mathcal{E}$ is

$$
W(t)=\operatorname{Var} \omega(t)=\frac{1}{K} \sum_{k=1}^{K}\left(\omega^{(k)}(t)\right)^{2}-\left(\frac{1}{K} \sum_{k=1}^{K} \omega^{(k)}(t)\right)^{2} .
$$

The average of a function $f(U)$ over $\mathcal{E}$ is defined as

$$
\langle f\rangle_{\mathcal{E}}=\frac{1}{K} \sum_{k=1}^{K} f\left(U^{(k)}\right) \omega^{(k)} .
$$

When $W(t)$ becomes too large, our aim is to transform $\mathcal{E}$ in a new ensemble $\mathcal{E}^{\prime}$ with a rule $\mathcal{E} \rightarrow \mathcal{E}^{\prime}$ such that the variance $W$ is reduced while the averages are kept constant. Actually, this is a formidable task, but we can achieve it at least approximately, namely in the $K \rightarrow \infty$ limit. To this aim, we extract $K$ independent integers $n_{1}, \ldots, n_{K}$ with $n_{i} \in\{1, \ldots, K\}$ and probabilities

$$
\operatorname{Prob}\left(n_{i}=k\right)=\frac{\omega^{(k)}}{\sum_{k^{\prime}=1}^{K} \omega^{\left(k^{\prime}\right)}}
$$

We then define $\mathcal{E}^{\prime}$ by

$$
\mathcal{E}^{\prime}=\left\{\left(U^{\left(n_{i}\right)}(t), 1\right)\right\}_{1 \leq i \leq K}
$$

In other words, we replace the information carried by the weights with that encoded in the multiplicities of unit weight walkers. By the large numbers law we expect

$$
\langle f\rangle_{\mathcal{E}}-\langle f\rangle_{\mathcal{E}^{\prime}} \stackrel{K \rightarrow \infty}{\longrightarrow} 0
$$


In actual calculations, there is therefore a systematic error which is removed by performing simulations with different values of $K$ and somehow extrapolating the $K \rightarrow \infty$ limit.

The calculation of observables when stochastic reconfiguration is in progress does not present any additional problem. The only point we stress is that, for a general operator $\Omega$, the weights at time $t_{1}+t_{2}$ are used to weight $\Omega\left(t_{1}\right)$ and therefore one must keep track for each reconfigured walker of its states at that past time (usually, a small number of updates is enough).

\section{ADAPTIVE OPTIMIZATION OF THE TRIAL WAVE FUNCTION}

Let us consider a trial wave function $\Psi_{0}(U, \mathbf{a})$ depending on some parameters $\mathbf{a}=\left(a_{1}, \ldots, a_{p}\right)$ and the GFMC calculation of an observable quantity that, for simplicity, we choose to be the ground state energy $E_{0}$.

We do not discuss the systematic error associated with $\varepsilon$ appearing in Eq. (2.17). The choice of $\varepsilon$ is related to the approximation involved in the operator splitting and also in the evaluation of the $\mathrm{SU}(\mathrm{N})$ heat kernel. A common model independent choice is to take $\varepsilon$ enough small to approximate the diffusion over $\mathrm{SU}(\mathrm{N})$ with that on the flat tangent space at the identity. Problems may then occur only if $\nabla F$ becomes too large, a constraint that can

be checked during the simulation. In the following we shall choose a reasonably small $\varepsilon$ and work under the hypothesis that the $\varepsilon \rightarrow 0$ extrapolation does not change our conclusions on the trial wave function dependence.

A simulation with a population of $K$ walkers will provide after $S$ Monte Carlo steps an approximate estimator $\hat{E}_{0}(S, K, \mathbf{a})$ of $E_{0}$. This is a random variable with the property

$$
\left\langle\hat{E}_{0}(S, K, \mathbf{a})\right\rangle=E_{0}+c_{1}(K, \mathbf{a}),
$$

and

$$
\operatorname{Var} \hat{E}_{0}(S, K, \mathbf{a})=\frac{c_{2}(K, \mathbf{a})}{\sqrt{S}}
$$

where $\langle\cdot\rangle$ denote the average over the Monte Carlo realizations. The finite population functions $c_{i}(K, \mathbf{a})$ satisfy

$$
\lim _{K \rightarrow \infty} c_{i}(K, \mathbf{a})=0
$$

where, by a self-averaging argument, it is reasonable to guess the asymptotic form $c_{2}(K, \mathbf{a}) \sim$ $\gamma(\mathbf{a}) / \sqrt{K}$ for large $K$. The average of $\hat{E}_{0}$ extrapolated at $K \rightarrow \infty$ is exact and independent on the trial parameters a. On the other hand, the function $c_{2}(K, \mathbf{a})$ may be strongly dependent on them. Since $c_{2}$ determines the statistical error at fixed computational time, it is important to minimize it.

If the family of functions $\Psi_{0}(U, \mathbf{a})$ includes the exact ground state at the special point $\mathbf{a}=\mathbf{a}^{*}$ then we know that

$$
c_{1}\left(K, \mathbf{a}^{*}\right)=c_{2}\left(K, \mathbf{a}^{*}\right)=0 .
$$

In a less optimal situation one has the two possibilities of minimizing $c_{1}$ or $c_{2}$. Motivated by [13], we choose to pursue the second alternative and seek a minimum of $c_{2}$. 
Moreover, we propose to optimize a adaptively. This can be done in the following way: the constant $c_{2}$ is related to the non zero fluctuations of $\widetilde{V}$ defined by

$$
\tilde{V}(U, \mathbf{a})=V(U)-\frac{1}{2}(\nabla F(U, \mathbf{a}))^{2}-\frac{1}{2} \nabla^{2} F(U, \mathbf{a}),
$$

where $\Psi_{0}(U, \mathbf{a})=\exp F(U, \mathbf{a})$. The ensemble variance of $\tilde{V}$ is a function of $\mathbf{a}$

$$
\operatorname{Var}_{\mathcal{E}} \tilde{V}=\frac{1}{K} \sum_{k=1}^{K} \tilde{V}\left(U^{(k)}, \mathbf{a}\right)^{2} \omega^{(k)}-\left(\frac{1}{K} \sum_{k=1}^{K} \tilde{V}\left(U^{(k)}, \mathbf{a}\right) \omega^{(k)}\right)^{2} .
$$

Our proposal is to update a according to the simple equation

$$
\mathbf{a}_{n+1}=\mathbf{a}_{n}-\eta \nabla_{\mathbf{a}} \operatorname{Var}_{\mathcal{E}} \tilde{V},
$$

where $\eta$ is a constant parameter and $\mathbf{a}_{n}$ is the value of $\mathbf{a}$ at the n-th update. In other words, following the spirit of [13], we implement a local minimization of the weight variance as a driving mechanism for the free parameters. It is important to remark that it is incorrect to look for the special parameters a that minimize $\operatorname{Var}_{\mathcal{E}} \widetilde{V}$ for a fixed ensemble $\mathcal{E}$. In fact, the equilibrium distribution of the link variables in the ensemble do depend on a and the addition of Eq. (4.7) to the Monte Carlo core establishes a non trivial feedback between the two sets of variables.

The coupled set of equations Eqs. (2.17,4.7) for the evolution of a and the random walkers is non linear and discrete. The above procedure is reasonable, but a test in explicit examples is required to check the convergence and stability of the method. A first analysis is described in [9] for a toy model with U(1) gauge symmetry in low dimensions. Here we consider the less trivial non Abelian four dimensional case.

\section{SIMULATION AND RESULTS}

\section{A. General Setup}

We simulate the $\mathrm{SU}(2)$ lattice gauge theory on a spatial $4^{3}$ lattice. The temporal extension is in principle infinite. Of course, the meaning of this statement is that in the Hamiltonian formulation we are projecting onto the exact ground state since we are applying many times an approximate form of $\exp (-\varepsilon H)$. In all our simulations $\varepsilon=0.01$. Another parameter that we keep fixed for simplicity is the number of elementary time evolutions between two stochastic reconfigurations. We choose 10 steps which is roughly the decorrelation time of the lattice configuration. A priori, a better choice of this parameter can improve the convergence of the $K \rightarrow \infty$ extrapolation, but we checked that in our range of parameters, the sensitivity on the choice of $K$ is quite small. We also fix the learning parameter $\eta=0.001$ for the adaptive determination of the wave function coefficients.

The parameters that remain free are therefore: the number $K$ of walkers, the number of coefficients in the ground state wave function and of course the coupling constant $g$ which is needed to discuss the possible scaling of the measured quantities. In more details, for each value $K=2,10,50,100$ and for each choice of the trial wave function we run the algorithm at five different coupling constants 


$$
\lambda=4 / g^{4}=0.8,0.9,1.0,1.1,1.2
$$

and measure the ground state energy $E_{0}$ and all the Wilson loops $W_{I J}$ with area $I J \leq 9$. We recall that the variable $4 / g^{4}$ is natural in the strong coupling expansion analysis.

\section{B. Adaptive Optimization}

The first topic that we discuss is the adaptive optimization of the free parameters $\mathbf{a}$ in the trial wave function. Motivated by the long wavelength strong coupling analysis of [8], we consider the following form of $F$

$$
F=c_{1} W_{1 \times 1}+c_{2} W_{(1 \times 1)^{2}}+c_{3} W_{1 \times 2, \text { planar }}+c_{4} W_{1 \times 2, \text { bent }},
$$

where $W_{1 \times 1}, W_{(1 \times 1) 2}, W_{1 \times 2 \text {,planar }}$ and $W_{1 \times 2 \text {,bent }}$ are the sum of all the plaquettes of the kind shown in Fig. 1. The coefficients $c_{1}, c_{2}, c_{3}$ and $c_{4}$ may be approximated as in [8] with a power series in $1 / g^{2}$ computed on the infinite lattice. Here, we exploit the dynamical system Eq.(4.7) to determine the finite size optimal $c_{i}$. In the analysis we discuss three specific scenarios that we characterize by the level $l=0,1$ or 4 , the number of free parameters:

$$
\begin{aligned}
& l=0: F \equiv 0, \text { no Importance Sampling; } \\
& l=1: c_{2}=c_{3}=c_{4}=0, \text { one free parameter; } \\
& l=4: \text { all } c_{i} \text { are optimized. }
\end{aligned}
$$

In Fig. 2, we show for example the evolution of $c_{1}$ with Monte Carlo time in the case $K=2,1 / g^{2}=0.5$ and $l=1$. The coefficients $c_{i}$ typically stabilize on values adaptively computed by the algorithm with small fluctuations that can be averaged in the long time limit. The initial values of $c_{i}$ for $K=10,50,100$ are taken from the run with the nearest smaller $K$ in order to reduce thermalization times. The procedure turns out to be stable at each considered $K$ and $g$. The fluctuations are bigger at smaller $g$, that is in the critical limit, while the equilibrium values agree reasonably well with the strong coupling approximation at large $g$. If the fluctuations were too noisy, then Stochastic Gradient Approximation techniques [14] could be applied by gradually reducing $\eta$ in Eq. (4.7).

\section{C. $K \rightarrow \infty$ extrapolation}

For each level $l$, we consider simulations with $K=2,10,50$ and 100 . The $K \rightarrow \infty$ limit does not depend on $l$, but the rapidity of the convergence yes. In Figs. (3-4) we plot the $K$ dependent estimates of $E_{0}$ and $W_{1 \times 1}$ for $l=0,1,4$ at $1 / g^{2}=0.5$. In the left plots we see clearly that for $l=0$ and $l=1$ the extrapolation $K \rightarrow \infty$ is largely uncertain. Unless we know the asymptotic behavior in $K$, we cannot safely determine the correct limit with the present data. A non rigorous guess is a power law behavior with finite $K$ corrections of the form

$$
c_{1}(K, \mathbf{a})=\frac{c_{1}(\mathbf{a})}{K^{\alpha(\mathbf{a})}}+o\left(K^{-\alpha}\right), \quad \alpha(\mathbf{a})>0
$$


that has been observed empirically [9]. Such behavior can be matched to the $K=10,50,100$ data, but its choice is rather arbitrary and, to be supported, would require more points at larger $K$. On the other hand, data computed in the $l=4$ scheme show a plateau beginning at $K \simeq 50$ and data at $K=100$ will therefore be used as an estimate of the $K \rightarrow \infty$ limit.

To check consistency of the runs at different $K$ and to evaluate quantitatively the improvement gained with larger $K$, we introduce an effective $K$

$$
K^{\prime}=K / \lambda(l)
$$

depending on the rescaling factor $\lambda(l)$. In the right plots of Figs. (3-4), we show how the curves for $E_{0}$ and $W_{1 \times 1}$ measured at different $K$ collapse on a single curve when shown as functions of $K^{\prime}$. The values of $\lambda(l)$ are

$$
\lambda(0)=1.6 \cdot 10^{3}, \quad \lambda(2)=5, \quad \lambda(4) \equiv 1 .
$$

The raw simulation, $l=0$, is very poor, as expected. On the other hand, the one parameter wave function gives the same accuracy of the $l=4$ one when the number of random walkers is roughly 5 times bigger.

We now determine the computational cost as a function of $l$. Let $n$ be the number of Monte Carlo iterations; the statistical error in the measure of a given observable and the CPU time are respectively given by

$$
\varepsilon_{\text {stat }}=\frac{\gamma_{l}}{\sqrt{n K}}, \quad t_{C P U}=\frac{n K}{v_{l}},
$$

where $\gamma_{l}$ and $v_{l}$ are normalization constants expected to be a decreasing function of $l$. Of course, $\gamma_{l}$ depends on the chosen observable. From Eq. (5.6) we deduce that

$$
t_{C P U}=\frac{\gamma_{l}^{2}}{v_{l}} \frac{1}{\varepsilon_{\text {stat }}^{2}}
$$

is independent on $K$. From our data, we can give the rough estimate of the ratios $v_{1} / v_{4}$ and $\gamma_{1} / \gamma_{4}$ when the coupling is $1 / g^{2} \simeq 0.5$. We find

$$
v_{1} / v_{4} \simeq 8, \quad \gamma_{1} / \gamma_{4} \simeq 2,
$$

and therefore $t_{C P U, l=4} \simeq 2 t_{C P U, l=1}$. Of course one must also remember that, as discussed above, $l=1$ does not show a clear plateau when $K<100$ and that very large $K$ cannot be used in $3+1$ dimensions because of storage limitations. Larger Wilson loops show a similar behavior with slightly better improvement in the case $l=4$.

In this particular model and at the considered volume and couplings we summarize the comparison between $l=4$ and $l=1$ at fixed systematic and statistical errors by saying that the improved wave functions are twice slower, but require a storage $\simeq 5$ times smaller. In the following, we shall pursue the $l=4$ choice because only in that case, our data can be safely considered asymptotic with respect to the $K \rightarrow \infty$ limit. 


\section{Scaling of the string tension}

The $l=4, K=100$ measurements of $E_{0}$, the Wilson loops with area $\leq 9$ and the coefficients $c_{i}$ are shown in Figs. (5-7) as functions of $1 / g^{2}$. The energy measurements agree well with the second order strong coupling expansion

$$
E_{0}=\frac{4}{g^{4}}-\frac{8}{3 g^{8}}+\mathcal{O}\left(\frac{1}{g^{12}}\right)
$$

An important check of the simulation requires an attempt to investigate scaling. The easiest observable quantity with simple Renormalization Group behavior is the string tension $\sigma$ that we obtain from Creutz's ratios

$$
\sigma=\lim _{I, J \rightarrow \infty} \sigma_{I, J}, \quad a^{2} \sigma_{I, J}=-\log \frac{W_{I, J} W_{I+1, J+1}}{W_{I, J+1} W_{I+1, J}} .
$$

This must be compared with the asymptotic scaling prediction

$$
a(g)=\frac{1}{\Lambda_{H}} f(g), \quad f(g)=\left(\frac{24 \pi^{2}}{11 g^{2}}\right)^{51 / 121} \exp \left(-\frac{12 \pi^{2}}{11 g^{2}}\right) .
$$

Even if we are work on a lattice with rather small spatial extension, it is interesting to compare our results with those obtained in the Lagrangian formulation. We choose the measurements in [15] as representatives of what can be obtained at values of the coupling in the range Eq. (5.1). In Fig. 8 we show $a \sqrt{\sigma_{1,2}}$ and $f(g)$. Loops larger than $2 \times 3$ cannot be used since in our computation they have too large errors.

The Lagrangian measurement is $\Lambda_{L}=(1.19 \pm 0.15) \cdot 10^{-2} \sqrt{\sigma}$. Taking into account the $\mathrm{SU}(2) \Lambda$ parameter ratio $\Lambda_{H} / \Lambda_{L}=0.84$ [16], we obtain the band shown in the Figure. As one can see, the Hamiltonian measurement is slightly larger, but reasonably consistent with the Lagrangian one.

\section{CONCLUSIONS}

The aim of this paper has been a study of the non perturbative behavior of the $S U(2)$ lattice gauge theory in $3+1$ dimensions by Hamiltonian Monte Carlo methods. Our results can be summarized into two main statements.

First, we have shown that the algorithm [9] is able to adaptively optimize the many parameters ground state trial wave functions needed to guide GFMC simulations of realistic models.

Second, we have discussed to what extent the use of improved wave functions is actually necessary, at least with the considered volume and couplings. From the purely computational point of view our data show that wave function improvement reduces the algorithm performance, but permits a substantial gain in storage. This means that we are allowed to work with a smaller number of walkers, $K$. This can be an important advantage, especially on larger lattices or at smaller couplings where the minimum $K$ at which a safe extrapolation to the $K \rightarrow \infty$ limit is feasible is expected to increase. 
As a non trivial check of the algorithm and of the strategy for the removal of the finite $K$ systematic error, we studied the string tension with a four parameter trial wave function and found a scaling behavior compatible with similar Lagrangian measurements.

Our investigation has been performed on a cluster of small computers and we could not attempt to measure glueball mass ratios. At this stage, the Hamiltonian formulation does not offer any special advantage with respect to the Lagrangian one. The storage gain associated to the absence of the temporal dimension is compensated by the memory required

to manage the walkers ensemble. In practice, we did not examine finite size scaling of the algorithm, a task that we leave for future studies. We stress however that many of the advanced techniques that are currently used in Lagrangian calculations [2], can be applied in the Hamiltonian formalism as well. Examples are the use of refined glueball operators or the improvement programme that has been recently extended to Kogut-Susskind Hamiltonians [17.

\section{ACKNOWLEDGMENTS}

I whish to thank Prof. G. Curci for many useful discussions on the numerical simulation of lattice field theories. 


\section{REFERENCES}

[1] J. B. Kogut and L. I. Susskind, Phys. Rev. D 11, 395 (1975);

J. B. Kogut, Rev. Mod. Phys. 51, 659 (1979).

[2] C. J. Morningstar and M. Peardon , Phys. Rev. D 56 , 4043 (1997);

C. J. Morningstar and M. Peardon, Phys. Rev. D 60, 034509 (1999).

[3] C. Long, D. Robson, S. Chin, Phys. Rev. D 37 , 3006 (1988).

[4] D. M. Ceperley and M. H. Kalos in "Monte Carlo Methods in Statistical Physics", ed. K. Binder, Springer-Verlag, Heidelberg (1992).

[5] C. J. Hamer, Nucl. Phys. B (Proc. Supp.) 47, 298 (1996).

[6] W. von der Linden, Phys. Rep. 220 , 53 (1992).

[7] M. Calandra Buonaura, S. Sorella, Phys. Rev. B 57 , 11446 (1998).

[8] S. Guo, Q. Chen, L. Li, Phys. Rev. D 49, 507 (1994).

[9] Matteo Beccaria, hep-lat/9907015, to appear on European Physical Journal C;

Matteo Beccaria, cond-mat/9912389, to appear on Physical Review D.

[10] P. Menotti and E. Onofri, Nucl. Phys. B 190, 288 (1981).

[11] S. A. Chin, O. S. van Roosmalen, E. A. Umland, S. E. Koonin, Phys. Rev. D 31, 3201 (1985).

[12] J. H. Hetherington, Phys. Rev. A 30 , 2713 (1984).

[13] C. J. Umrigar, K. G. Wilson, J. W. Wilkins, Phys. Rev. Lett. 601719 , 88 (19.)

[14] A. Harju, B. Barbiellini, S. Siljamäki and R. M. Nieminen, Phys. Rev. Lett. 79, 1173 (1997).

[15] B. Berg, A. Billoire, C. Rebbi, Ann. Phys. 142, 185 (1982).

[16] A. Hasenfratz, P. Hasenfratz, Nucl. Phys. B 193, 210 (1981).

[17] X. Luo and S. Guo , Phys. Rev. D 59 , 034503-1 (1999). 


\section{FIGURES}

FIG. 1. Plaquettes appearing in the $l=4$ trial wave function. A sum over the lattice is understood.

FIG. 2. Monte Carlo history of the trial wave function coefficient $c_{1}$ at $1 / g^{2}=0.5$ and $K=2$. The initial state of the system is cold.

FIG. 3. Dependence of $E_{0}$ on $K$ with and without reparametrization $K \rightarrow K^{\prime}$ for the three schemes $l=0, l=1$ and $l=4$. The coupling constant is $1 / g^{2}=0.5$.

FIG. 4. As Fig. 3, but for the $1 \times 1$ Wilson loop.

FIG. 5. Coupling constant dependence of $E_{0}$ at $l=4, K=100$.

FIG. 6. Coupling constant dependence of the trial wave function coefficients at $l=4, K=100$.

FIG. 7. Coupling constant dependence of the Wilson loops at $l=4, K=100$.

FIG. 8. Scaling plot of the string tension. Comparison between $a \sqrt{\sigma_{1,2}}$ and the asymptotic Renormalization Group prediction. The band corresponds to the Lagrangian measurement as explained in the text 
$\Omega$

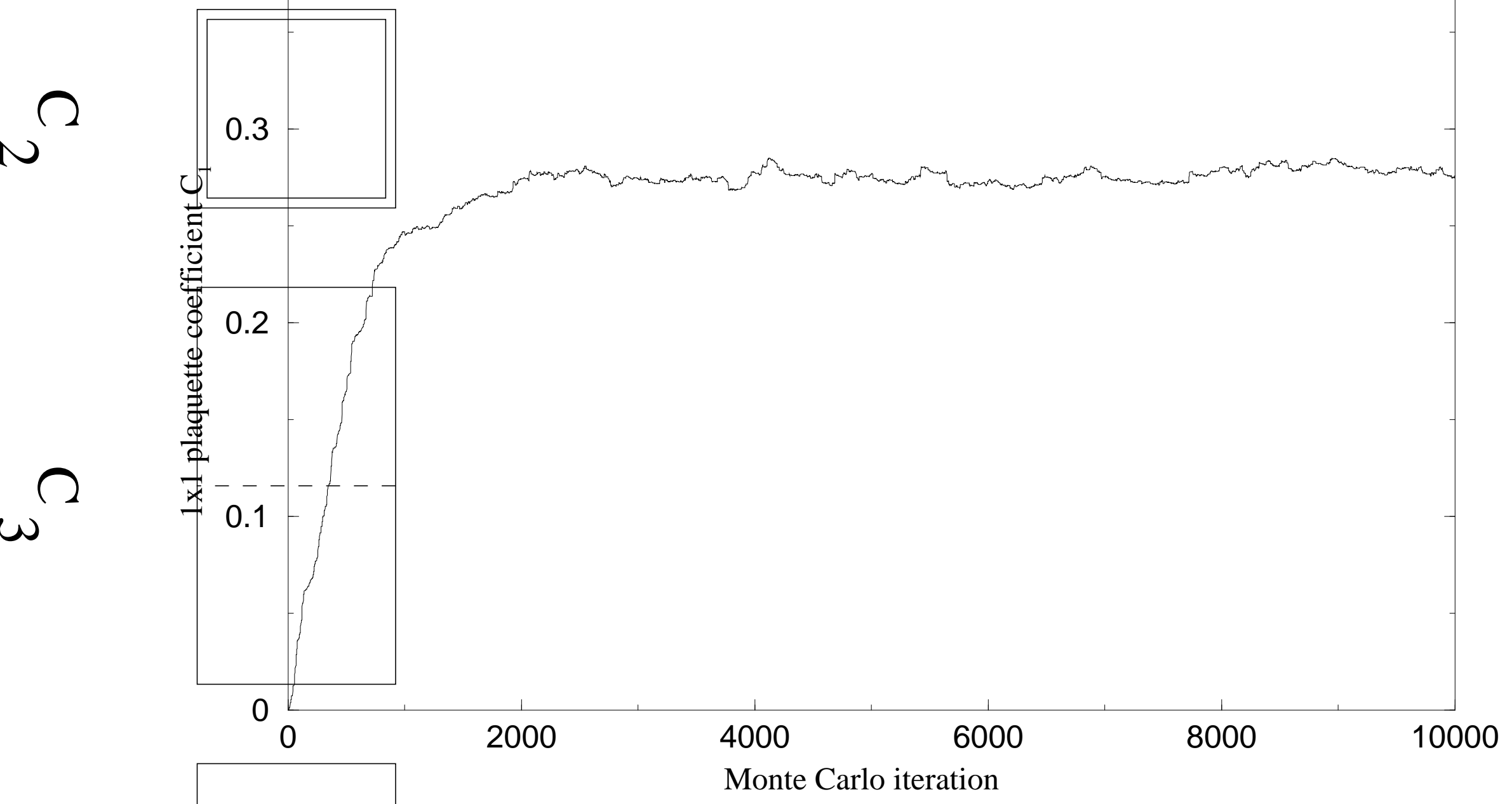




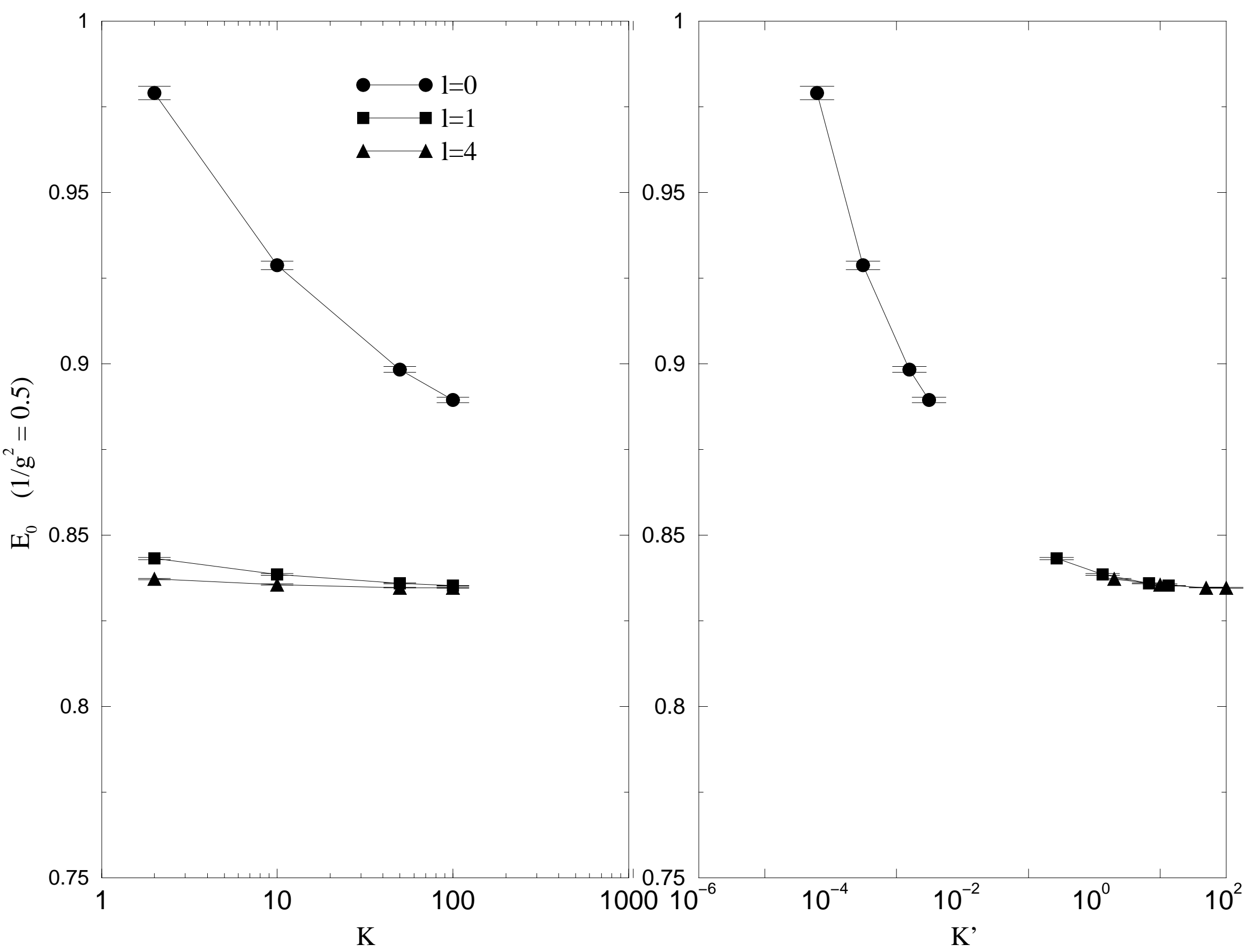




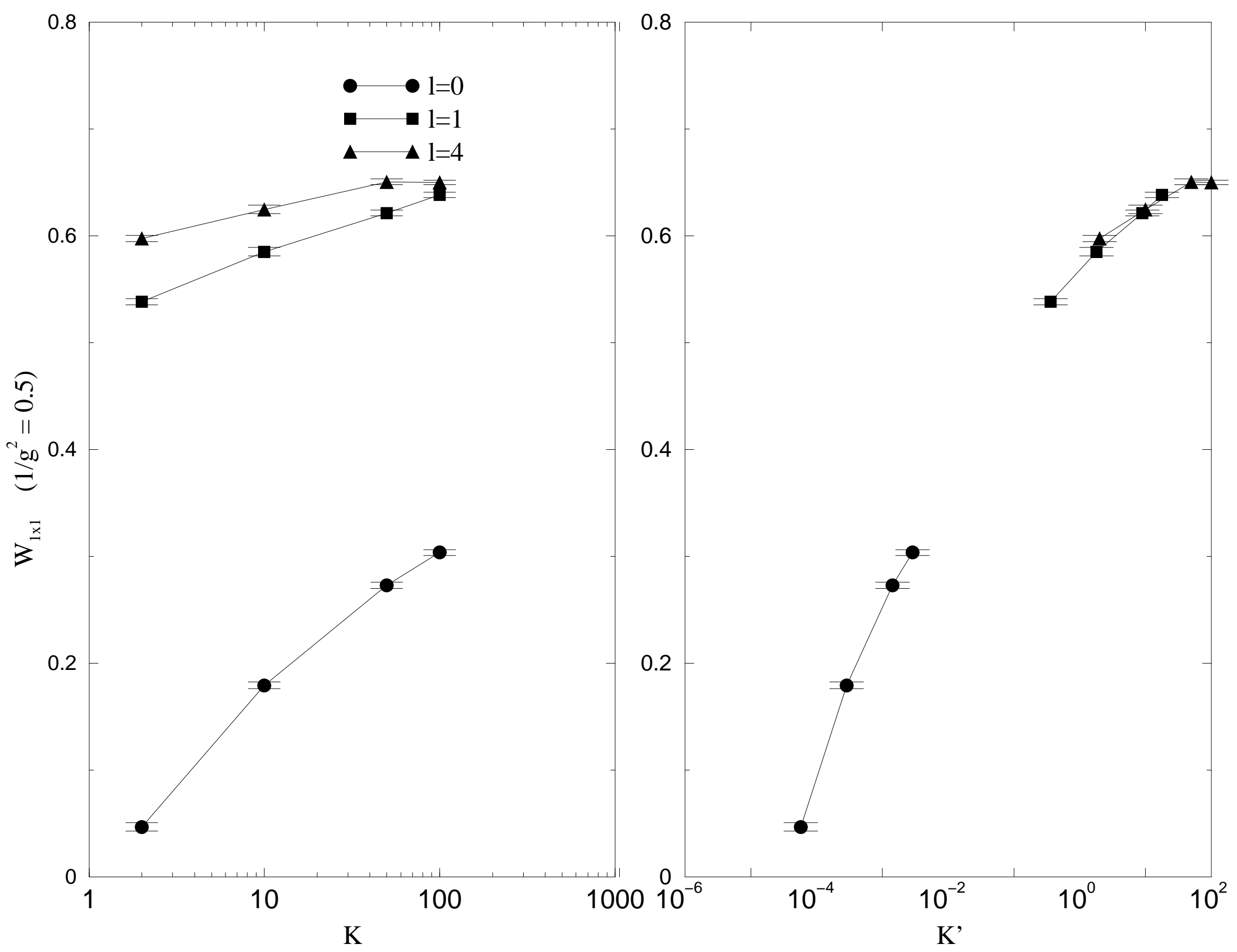




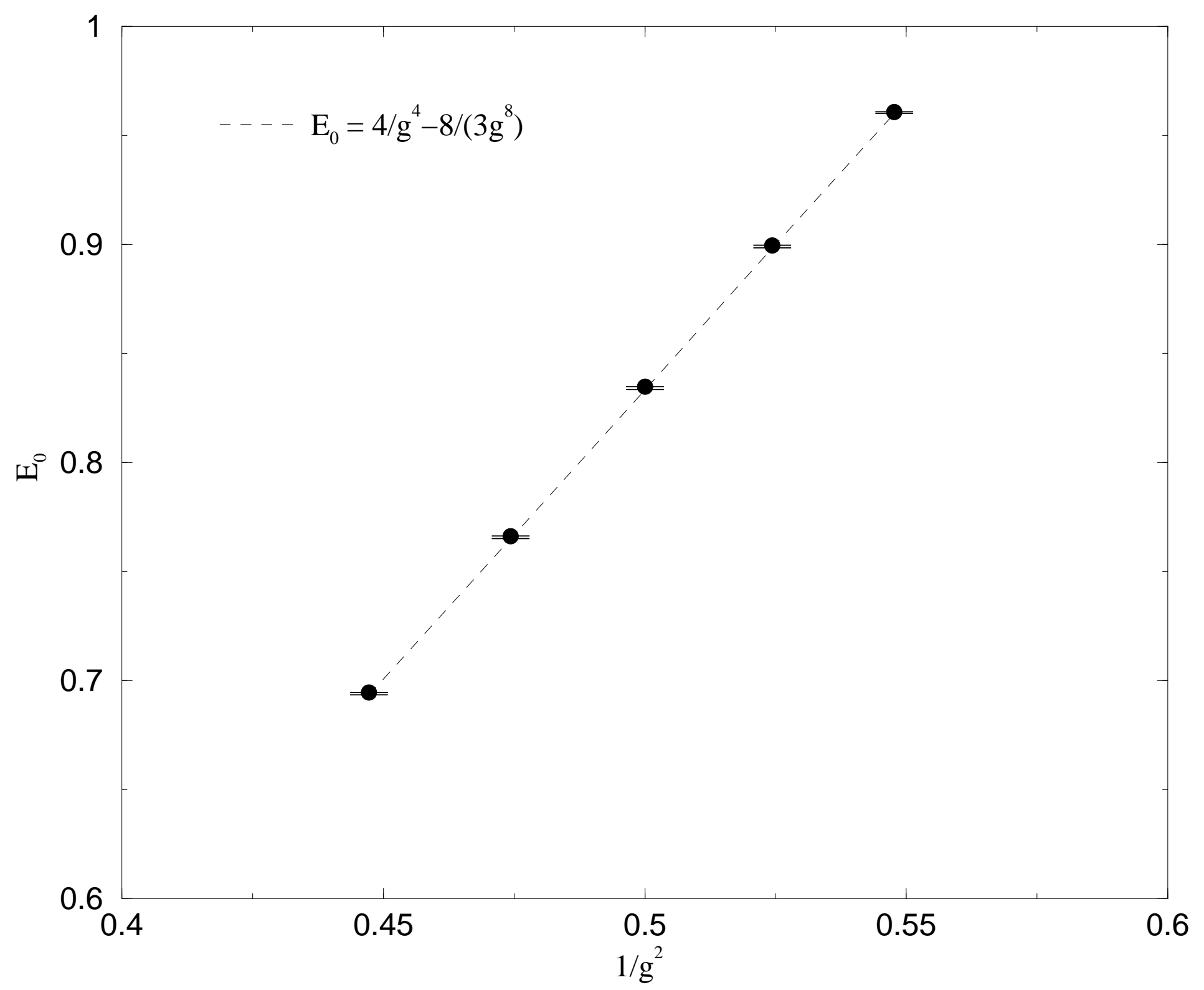




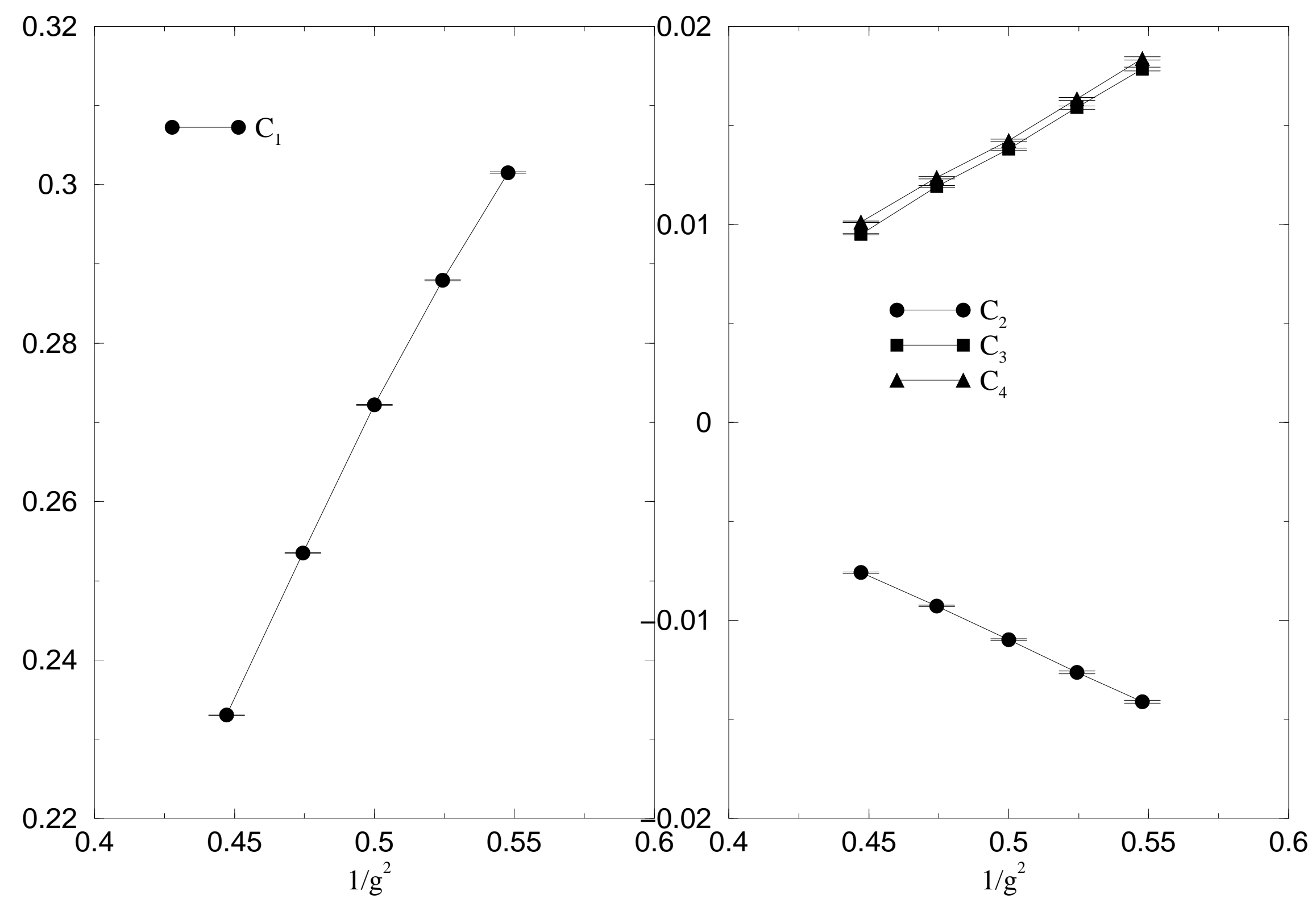




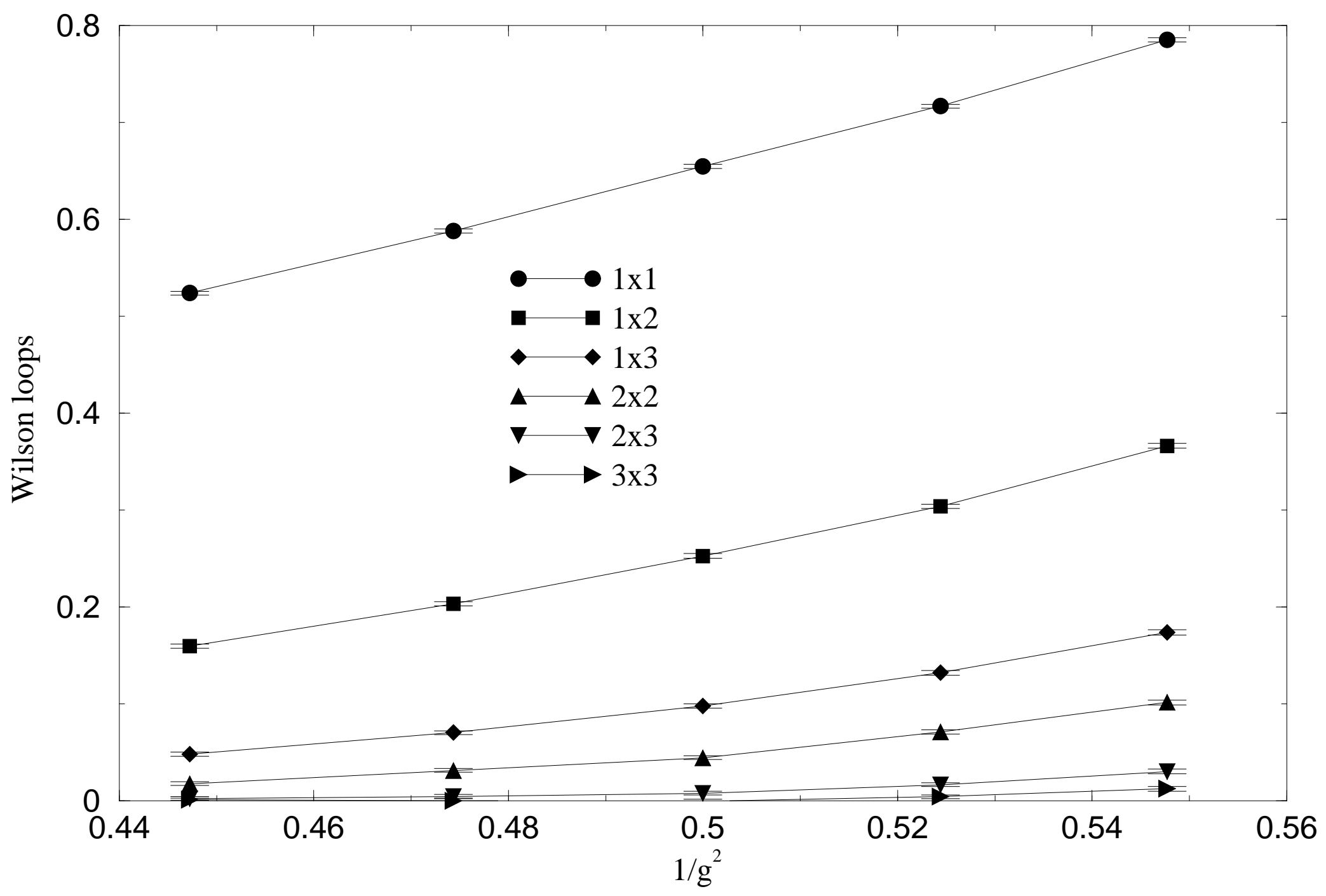




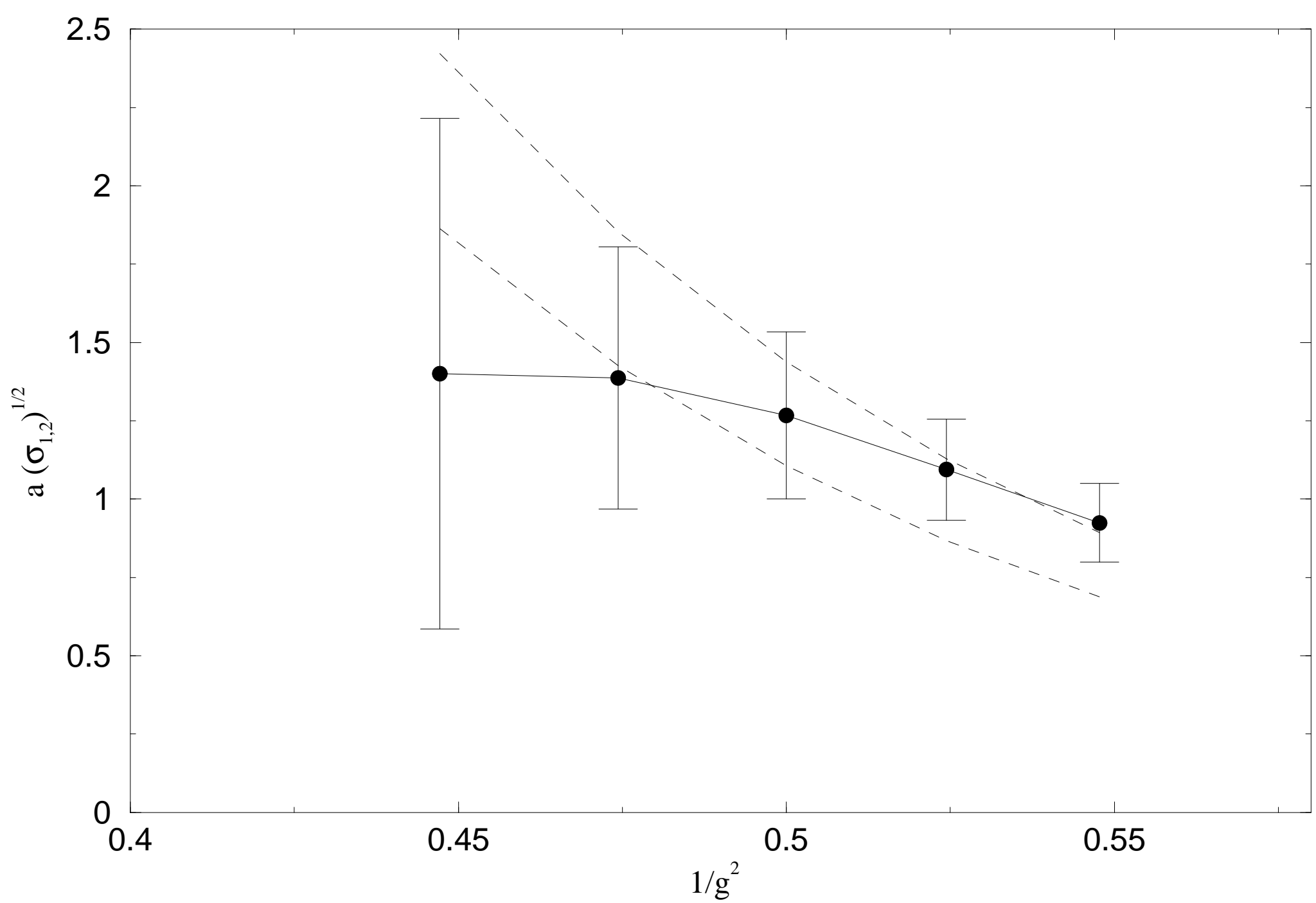

